DOE/ER/60674-5

\title{
BASE SEQUENCE EFFECTS ON INTERACTIONS OF AROMATIC MUTAGENS WITH DNA
}

\section{Progress Report}

for the period September 1, 1991 - August 31, 1992

N.E. Geacintov

New York University

New York, New York 10003

\section{NOTICE}

This report was prepared as an account of work supported by the United States Government. Neither the United States nor the United States Department of Energy, nor any of their contractors, subcontractors, or their employees, makes any warranty, express or implied, or assumes any legal liability or responsibility for the accuracy, completeness, or usefulness of any information, apparatus, product or process disclosed or represent that its use would not infringe privately owned rights.

September 30, 1992

Prepared for

THE U.S. DEPARTMENT OF ENERGY

AGREEMENT NO. DE-FGO2-88ER60674 


\section{PROJECT SUMMARY}

The chemical binding of bulky, mutagenic and carcinogenic polynuclear aromatic compounds to certain basesequences in genomic DNA is known to inhibit DNA replication, and to induce mutations and cancer. In particular, sequences that contain multiple consecutive guanines appear to be hot spots of mutation. The objectives of this research are to determine how the base sequence around the mutagen-modified target bases influences the local DNA conformation and gives rise to mispairing of bases, or deletions, near the lesion. Oligonucleotides containing one, two, or three guanines were synthesized and chemically reacted with the mutagen anti-7,8-dihydroxy-9,10-cpoxy-benzo[a]pyrene (BrDE), one of the most mutagenic and tumorigenic metabolites of benzo[a]pyrene. Adducts are formed in which only one of the guanines is modified by trans or cis addition to the exocyclic amino group. The BPDE-oligonucleotides are separated chromatographically, and the site of modification is established by Maxam-Gilbert high resolution gel electrophoresis techniques. The thermodynamic properties of duplexes using complementary, or partially complementary strands were examined. In the latter, the base opposite the modified guanine was varied in order to investigate the probability of mispairing of the modified $G$ with $A, T$ and $G$. The relative thermodynamic stabilities of these duplexes suggests that $A$ mispairs preferentially with the modified $G$ in (+)-trans-BPDE-oligo adducts, and with $T$ in (-)-transBPDE-oligo adducts. These results suggest that (+)-BPDE should preferentially give rise to G.C -..-> T.A transversions, as is indeed observed experimentally by other workers. In contrast, this speculative model suggests that the (-)enantiomer of BPDE should give rise preferably to G.C --.-> A.T transitions. This prediction remains to be tested. The successful synthesis of stereospecific and site-specific mutagen-oligonucleotide adducts opens new possibilitics for correlating adduct structure-biological activity relationships, and thus lead to a belter understanding of base-sequence effects in mutagenesis induced by energy-related bulky polynuclear aromatic chemicals. 


\section{A. INTRODUCTION AND OVERVIEW}

This report is a summary of research performed during the period September 1, 1991 to August 31, 1992 supported by DOE Grant No. DE-FGO2-88ER60674, and covers the second year of this three-year grant cycle which expires on February 28, 1994.

\section{OBJECTIVES}

The importance of base sequence in carrying genetic information is well known. DNA sequence data, under the human genome project, is rapidly accumulating and will ultimately lead to a detailed understanding of the role of base sequence in human disease. The chemical binding of mutagenic and tumorigenic metabolites of polynuclear aromatic hydrocarbon $(\mathrm{PAH})$ derivatives to certain genomic sequences can give rise to mutations and cancer. Sequences that contain multiple guanines (G's) appear to be hot spots of mutation and therefore play a special role in these phenomena. The objectives of this research are to systematically determine how the secondary structure of DNA, and the sequences of bases around the modified DNA base, affect the reactivities of PAH diol epoxide metabolites, and the properties of the carcinogen-DNA lesions which are formed. Our approach is based on the synthesis of site-specific and stereochemically defined adducts derived from monomeric and oligomeric deoxyoligonucleotides of defined base composition and sequence. A variety of spectroscopic and thermodynamic methods are utilized to characterize the interactions of bulky mutagenic lesions with oligonucleotides of defined base composition and sequence, and the concomitant alterations in the DNA conformation. Molecular modeling techniques, employing a range of available software, are being utilized to correlate the experimental results with energy-minimized molecular views of the PAH metabolites bound to different sequences in nucleic acids.

\section{SUMMARY OF PROGRESS WITHIN THE LAST YEAR}

I. Synthesis of Mutagen-Modified DNA Mini-Helices. Mutations induced by benzo[a]pyrene diol epoxide (BPDE), the most mutagenic metabolite of the energy-related pollutant benzo[a]pyrene, occur in genomic sequences which contain runs of two, three, or more guanines. Within the last grant year, we have focussed our efforts on synthesizing mini-DNA segments in which covalently bound BPDE lesions are selectively placed on one of these guanines in runs of two or three adjacent guanines. These BPDE-modified mini-DNA fragments can be complexea with complementary unmodified oligonucleotide strands to form model mini-double helices in which the placement and stereochemistry of the adducts are defined. These modified DNA segments are structurally characterized in our 
laboratory, and subsequently supplied to colleagues at other Universities who perform site-directed mutagenesis and other studies to elucidate structure-function relationships.

II. Thermodynamic Studies of Base-Mispairing in BPDE-modified DNA segments. Our ability to synthesize modified mini-DNA segments which contain mutagenic BPDE lesion placed at will at one of the bases, opens up new possibilities for adduct structure-function studies in mutagenesis, both on physico-chemical and biological levels. According to prevailing views, during DNA replication, the insertion of the incorrect base opposite the mutagenmodified base in the template strand leads to base substitution mutations. Frameshift mutations occur when the replication enzymes skip one or more bases near the modified base on the template strand; this process is believed to be facilitated when the carciongen-modified guanine assumes a bulge-like conformation at the site of fixation. In an effort to model such interactions, we have studied the thermodynamics of base pairing using BPDE-modified oligonucleotide and modified complementary strands. While the model systems used are not exactly the same as those which occur in real life situations at replication forks, they nevertheless constitute a first step in studies of the thermodynamic features and stabilities of structures relevant to this important biological phenomenon.

In this progress report we stress recent results dealing with these topics. We begin with a short intrductory background section relevant to the research results described here.

\section{B. BACKGROUND}

The ultimate mutagenic and carcinogenic metabolites of benzo[a]pyrene are the bay region diol epoxides [1]. There are two diastereomers, and each of these is resolved into a pair of enantiomers. The two (+) and (-) (or R,S,S,R and $S, R, R, S)$ enantiomers of the anti diastereomer trans-dihydroxy-anti-9,10-epoxy-7,8,9,10-tetrahydrobenzo[a]pyrene (BPDE) are of particular interest for structure-biological activity correlation studies because (+)-BPDE is highly tumorigenic while the (-) enantiomer is not (reviewed in [1]). We have recently succeeded in synthesizing stereospecific BPDE-oligonucleotide adducts by direct synthesis methods [2,3]. Both (+)- and (-)-BPDE give rise to $\mathrm{N}^{2}$-guanine-C10 BPDE adducts via trans and cis addition of $\mathrm{dG}$ relative to the 9,10-oxirane moiety of BPDE $[2,4]$. The stereochemical features of adduct formation are depicted in Fig. 1. Because the conformation of the cis and trans $-\mathrm{BPDE}-\mathrm{N}^{2}$-dG (deoxyguanine adducts) are structurally (4) and conformationally (3) so different from one another, it is expected that the biological characteristics and endpoints of these lesions will also be different $(3,4)$. Recently, we have succeeded in characterizing the solution structures of the (+)-trans- (5), (-)-trans- (6), and (+)-cis-BPDE- $\mathrm{N}^{2}-\mathrm{dG}$ adducts (7) in the double-stranded oligonucleotide sequence (C opposite the modified G, "natural" complementary strand): 


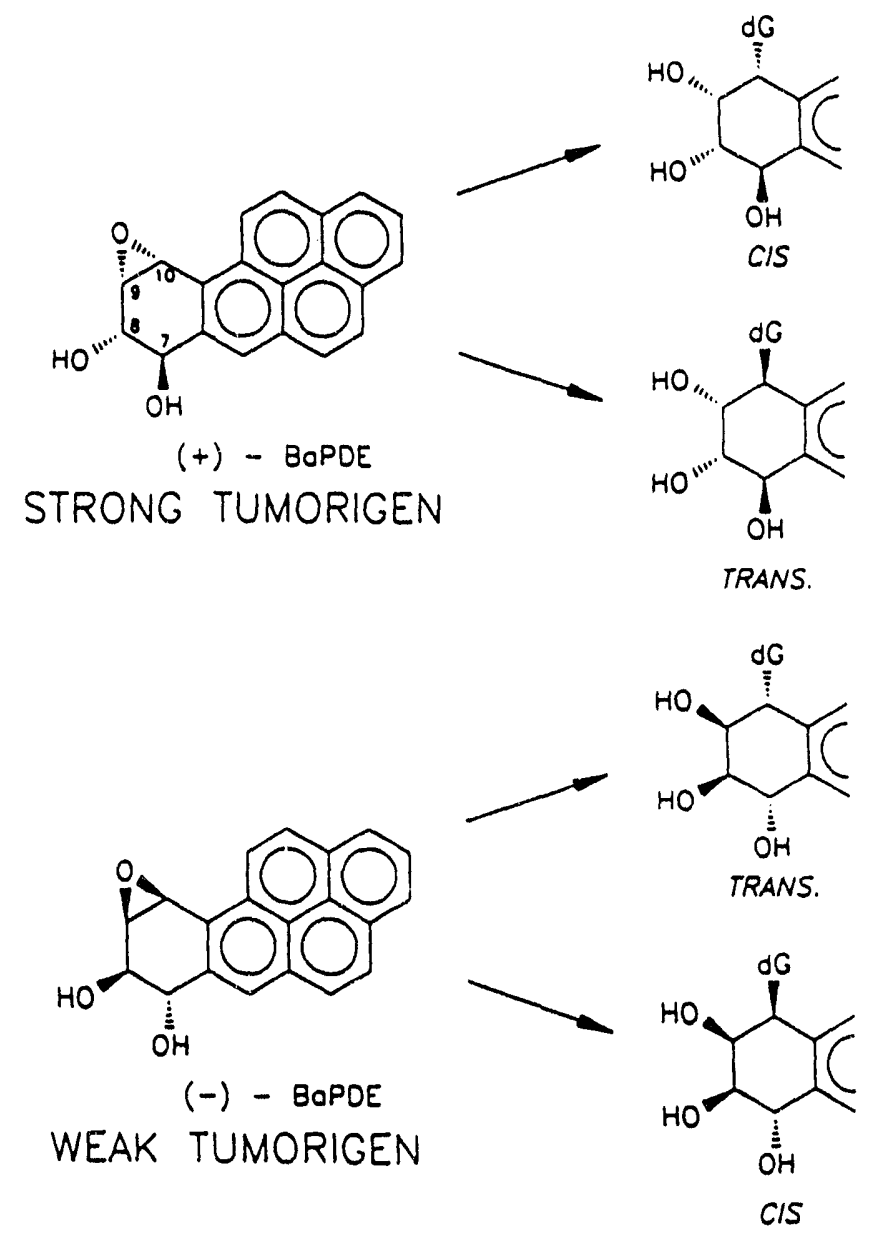

Fig. 1. Structures of BPDE stereoisomers and their $\mathrm{N}^{2}$ guanine adducts. 


$$
\begin{aligned}
& 5^{\circ} \text {-d(CCATCG }{ }^{\text {PPDE }} \text { CTACC) }-3^{\prime} \\
& 3^{\circ} \text {-d(GGTAGC......GATGG)-5' }
\end{aligned}
$$

(modified double-stranded sequence No. I)

In this report, we describe the thermodynamic melting and other parameters of sequence I, and similar double-stranded double-helices in which the base $\mathrm{X}$ opposite the modified $\mathrm{G}$ is mismatched (incorrect Watson-Crick base pairing), or is missing entirely. Specifically, $\mathrm{X}$ in the complementary strand can be either $\mathrm{C}, \mathrm{T}, \mathrm{A}$, or $\mathrm{G}$, or may be missing entirely as in the complentary strand $5^{\circ}-\mathrm{d}\left(\right.$ GGTAGGATGG) $-3^{\circ}$.

\section{MATERIALS AND METHODS}

The (+)- and (-)-BPDE enantiomers were synthesized according to published methods [8]. The oligonucleotides were synthesized using the phosphoramidite method, purified, reacted with BPDE, and the BPDE-oligonucleotide adducts were purified as described earlier [2,3]. Duplexes were produced by mixing equimolar amounts of the modified or unmodified single-stranded oligonucleotides and the complementary (or near-complementary) strands, heating to $70{ }^{\circ} \mathrm{C}$ for about 10 minutes, and then allowing the samples to cool slowly back to room temperature overnight. The thermodynamic characteristics of DNA duplex formation are obtained by studying the "melting curves." These are defined as the temperature-dependent changes in the absorbance of solutions of double-helical DNA measured near the absorption maximum. As the temperature is increased, the duplexes dissociate to form single-stranded DNA, and the absorbance increases (hyperchromicity). Absorbances were measured at $260 \mathrm{~nm}$ while the temperature was increased at a rate of $0.42{ }^{\circ} \mathrm{C} / \mathrm{min}$. The melting point $T_{m}$ is defined as the temperature at which $\alpha$, the fraction of strands in the duplex state, is equal to 0.5 [9]. However, $T_{m}$ is often difficult to determine because of poorly defined baselines. Therefore, $T_{m}$ was determined from the maxima in plots of the first order derivative, $d \alpha / d T$, versus the temperature $T$. The values of $T_{m}$ determined by these two methods are generally not identical, but are typically within 1-2 degrees of each other [10]. Experimental $T_{m}$ values obtained by the derivative method were reproducible to within $\pm 0.5{ }^{\circ} \mathrm{C}$, as determined from heating, cooling, and reheating cycles. The concentrations of the unmodified oligonucleotide strands were determined by the methods described by Warshaw et al. [11]. All samples were dissolved in $20 \mathrm{mM} \mathrm{Na}_{2} \mathrm{HPO}_{4}, 0.1 \mathrm{M} \mathrm{NaCl}$, pH 7.0 buffer solution.

For non-self complementary oligonucleotides in equilibrium:

$$
\text { [single strand] }+[\text { complement }]<\ldots . . . . .>\text { [duplex }]
$$


an equilibrium constant $\mathrm{K}$ can be defined which is related to the thermodynamic parameters of duplex formation via the usual equations

$$
\Delta G^{\circ}=-\mathrm{RT} \ln \mathrm{K}
$$

and

$$
\Delta G^{\circ}=\Delta H^{\circ}-T \Delta S^{\circ}
$$

The thermodynamic parameters can be derived from the $T_{m}$ values at a number of different strand concentrations $\left(C_{T}\right)$ from plots of $1 / T_{m}$ vs. $\ln C_{T}$ according to the equation:

$$
1 / \mathrm{T}_{\mathrm{m}}=\left(\mathrm{R} / \Delta \mathrm{H}^{\circ}\right) \ln \mathrm{C}_{\mathrm{T}}+\left(\Delta \mathrm{S}^{\circ}-0.83 \mathrm{R}\right) / \Delta \mathrm{H}^{\circ}
$$

as described in detail elsewhere [12]. The values of the molar enthalpies $\left(\Delta \mathrm{H}^{\circ}\right)$ and entropies $\left(\Delta \mathrm{S}^{\circ}\right)$ are obtained from an evaluation of the slopes and intercepts of such linear plots.

\section{THERMODYNAMICS OF HELIX-COIL TRANSITIONS IN STEREOSPECIFIC BENZO[a]PYRENE DIOL EPOXIDE-OLIGONUCLEOTIDE ADDUCTS.}

Our initial efforts are directed towards studying sequence I, since the solution structure of this particular doublestranded sequence has been clearly established by high-resolution NMR methods [5-7]. We studied the melting characteristics of the related double-stranded sequences:

$$
\begin{aligned}
& 5^{\prime}-\mathrm{d}\left(\mathrm{CCATCG}{ }^{\mathrm{BPDE}} \mathrm{CTACC}\right) \\
& 3^{\prime} \text {-d(GGTAGX......GATGG)-5' }
\end{aligned}
$$

(modified duplex No. II)

were $X=C, T, A, G$ or is missing entirely. The latter corresponds to a partially mismatched complementary 10-mer strand which is one base shorter than the modified oligonucleotide $5^{\prime}-\mathrm{d}\left(\mathrm{CCATCG}^{\mathrm{BPDE}} \mathrm{CTACC}\right)$. A typical melting profile is shown in Fig. 2. The melting points of modified and unmodified duplexes are dependent on the total strand concentration, $C_{T}$. Typical plots of $T_{m}$ vs. $\ln C_{T}$ are shown in Fig. 3. The values of $T_{m}$ and the thermodynamic parameters $\Delta \mathrm{G}^{\circ}, \Delta \mathrm{H}^{\circ}$, and $\Delta \mathrm{S}^{\circ}$, derived from these plots, are summarized in the tables below. 
1. Mismatched Bases opposite the modified G's $(X=T, A$, or $G)$

The values of the thermodynamic parameters $\Delta \mathrm{H}^{\circ}$ and $\Delta S^{\circ}$ for these "mismatched" duplexes II, in which there is only one incorrect base $\mathrm{X}$ in the unmodified complementary strand opposite the modified $\mathrm{G}$, are summarized in Tables I and II, respectively. The values of melting points $T_{m}$ evaluated from experimental data at strand concentration of $10^{-5} \mathrm{M}$, is summarized in Table III. The analogous values for the modified duplex I with $\mathrm{X}=\mathrm{C}$, are shown for comparison; the structure of this duplex is shown below:

$$
\begin{aligned}
& 5^{\circ}-\mathrm{d}(\text { CCATCGCTACC) } \\
& 3^{\circ}-\mathrm{d}\left(\text { GGTAGCGATGG) }-5^{\prime}\right.
\end{aligned}
$$

(unmodified natural duplex No. III)

We have not yet studied the related unmodified and mismatched duplexes

$$
\begin{aligned}
& \text { 5'-d(CCATCGCTACC) } \\
& \text { 3'-d(GGTAGXGATGG)-5' }
\end{aligned}
$$

(unmodified duplexes No. IV)

\begin{tabular}{|c|c|c|c|c|}
\hline & $\mathrm{X}=\mathrm{C}^{*}$ & $X=T$ & $X=A$ & $X=G$ \\
\hline unmodified (III) & $-95 \pm 5$ & -...-..- & (n.......... & -......... \\
\hline (+)-trans adduct (II) or (I) & $-84 \pm 5 \quad(I)$ & $-52 \pm 3$ & $-68 \pm 3$ & $-48 \pm 3$ \\
\hline$(-)$-trans adduct $(\mathbf{I I})$ or $(\mathbf{I})$ & $-67 \pm 4 \quad$ (I) & $-70 \pm 5$ & $-61 \pm 3$ & $-55 \pm 3$ \\
\hline
\end{tabular}

with $\mathrm{X}=\mathrm{T}, \mathrm{A}$, and $\mathrm{G}$; the corresponding positions in Tables I - III are therefore empty. These studies are presently still in progress.

Table I. Molar enthalpy values, $\Delta \mathrm{H}^{\circ}$ (kcal/mole) for duplex types I, II and III.

*Duplexes with natural complementary strand (C opposite the modified or unmodified $G$ ). 
Table II. Molar entropy values, $\Delta S^{\circ}$ (kcal/mole/deg) for duplex types I, II and III. All values within $\pm 10 \%$.

\begin{tabular}{||l|c|c|c|c|}
\hline & $X=C^{*}$ & $X=T$ & $X=A$ & $X=G$ \\
\hline \hline unmodified (III) & -0.21 & $\cdots-\cdots$ & $\cdots \cdots$ & $\cdots$ \\
\hline (+)-trans adduct (II) or (I) & -0.24 (I) & -0.14 & -0.20 & -0.13 \\
\hline$(-)$-trans adduct (II) or (I) & -0.19 (I) & -0.20 & -0.17 & -0.15 \\
\hline
\end{tabular}

*Duplexes with natural complementary strand (C opposite the modified or unmodified $\mathrm{G}$ ).

Table III. Melting points, $T_{m}$, evaluated at strand concentrations $C_{T}=10^{-5} \mathrm{M}$.

\begin{tabular}{|c|c|c|c|c|}
\hline & $\mathrm{X}=\mathrm{C}^{*}$ & $X=T$ & $X=A$ & $X=G$ \\
\hline unmodified (III) & 50.5 & $\ldots$ & -......... & -........ \\
\hline$(+)$-trans adduct (II) or (I) & $41.0 \quad$ (I) & 37.3 & 35.3 & 36.5 \\
\hline$(-)$-trans adduct (II) or (I) & 43.2 (I) & 35.9 & 32.4 & 33.1 \\
\hline
\end{tabular}

*Duplexes with natural complementary strand (C opposite the modified or unmodified G).

The conclusions which can be reached from these results are summarized below.

Natural Complentary strands $(X=C)$.

It is immediately evident from the second column in Table III that the presence of the covalently bound ( + )and (-)-BPDE residues in the trans configuration destabilizes the double helix by 7 - 9 degrees (at a strand concentration of $10^{-5} \mathrm{M}$ ). From solution NMR studies, it is known that the hydrophobic and bulky pyrenyl residue are situated in a somewhat widened minor groove of a B-DNA like structure in both cases [6]. The slightly extended phosphate diester backbone must result in weakened base-base stacking interactions near or at the binding sites. Indeed, the smaller enthalpy of melting values (Table I) may well reflect a difference in the interactions between the planar DNA bases. 


\section{Trans (-) \#91-102, $260 \mathrm{~nm}(10)$ Melting Clive (6.19.92, S.Smirnov)}

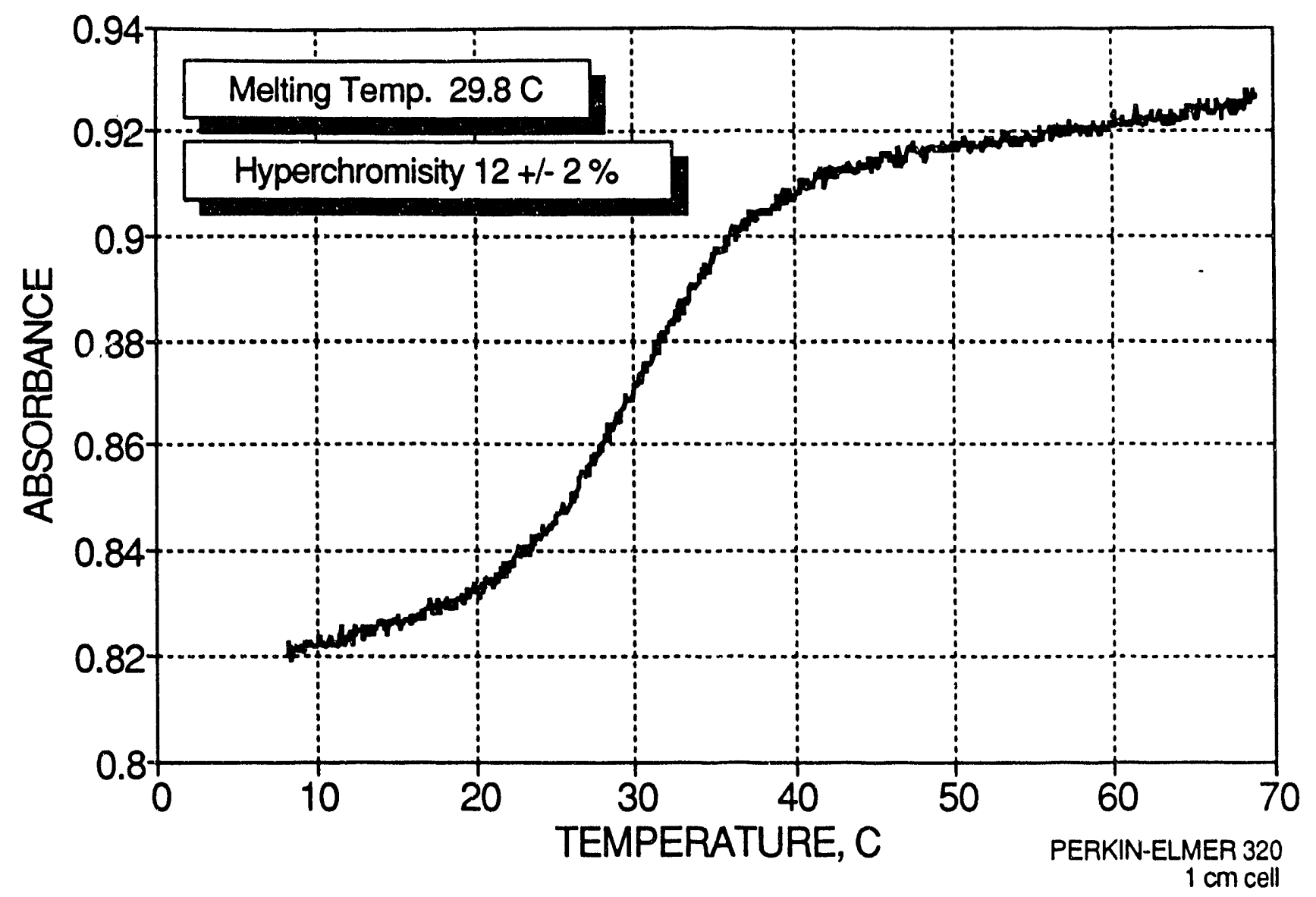

Fig. 2. Typical meiting profile, duplex type II with A-mismatch. 


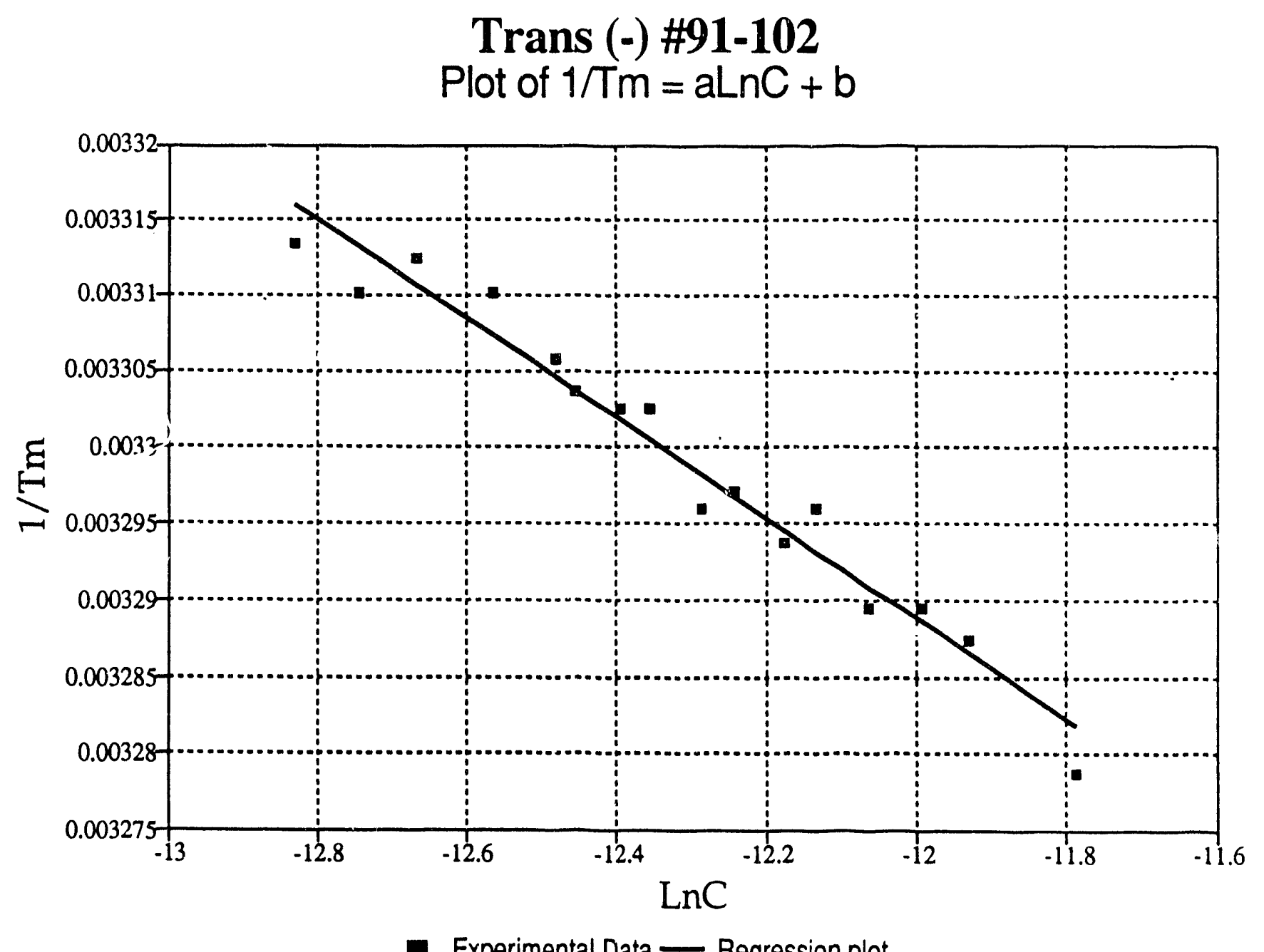

- Experimental Data - Regression plot

Fia. 3. A typical concentration plot (melting point vs. DNA strand concentration). Duplex type II with A-mismatch. 
The differences between the molar entropies of duplex formation are only slightly smaller for the adducts than for the unmodified duplex (Table I). A larger negative change signifies a greater degree of order in the duplex relative to the two separated strands. Indeed, the values of $\Delta S^{\circ}$ for duplex formation in the case of the two BPDE-modified strands are less negative than in the case of the unmodified strand (Table II). However, in view of the experimental errors involved, these differences are not pronounced. In summary, the BPDE-modified duplexes are less stable than the unmodified duplexes as shown by the melting point values in Table III. The (-)-trans-adduct exhibits smaller absolute values of $\Delta \mathrm{H}^{\circ}$ and $\Delta \mathrm{S}^{\circ}$ than the (+)-trans adduct (Tables I and II), yet its melting point is unexpectedly somewhat larger.

Single Base Mismatched Complements with $X=T, A$, and $G$.

One mechanism of base substitution mutation involves the pairing of an incorrect base with the BPDE-modified guanine on the template strand. If pairing occurs with $\mathrm{A}$ instead of a $\mathrm{C}$, then the observed mutations will be G.C -...-..-> T.A transversions. In the case of racemic ( \pm )-BPDE, the major point mutations in a variety of biological systems are G.C -.....-> T.A transversions [see, for example, ref. 13]. It is therefore of interest to examine the relative thermodynamic stabilities of duplexes with mismatched bases in the complementary strand opposite the BPDE-modified G. While these experiments do not exactly mimic events at replication forks, in which a single complementary base pairs with $G$, such experiments may nevertheless provide insight into the nature of events taking place.

The results for mismatched type III duplexes are summarized in tables I - III. It is evident that the melting points of all of the mismatched duplexes are lower by some $4-10^{\circ} \mathrm{C}$ than the duplexes with the natural complementary strands $(X=C)$. Especially in the case of the $(+)$-trans adduct, there is a significant decrease in $\Delta H^{\circ}$ by about 32 $36 \mathrm{kcal} /$ mole when the non-complemetary base is $\mathrm{T}$ or $\mathrm{G}$. However, when $\mathrm{X}=\mathrm{A}$, the decrease in the magnitude of $\Delta \mathrm{H}^{\circ}$ is only $\approx 16 \mathrm{kcal} / \mathrm{mole}$ (Table I). The corresponding entropy changes suggest that there is more order in the mismatched (+)-trans-duplex when $\mathrm{X}=\mathrm{A}$, than when $\mathrm{X}=\mathrm{T}$ or $\mathrm{G}$ (Table II). These effects, particularly the more negative $\Delta \mathrm{H}^{\circ}$ value, could be due to base-carcinogen stacking interactions between the planar pyrenyl residues and the mismatched adenine base. This mechanism is consistent with the largest negative entropy effect observed $(-0.20$ $\mathrm{kcal} / \mathrm{mol} / \mathrm{deg}$, Table II) upon duplex formation when $\mathrm{X}=\mathrm{A}$, rather than $\mathrm{T}$ or $\mathrm{G}$. Effects of this type may well rationalize the experimental observations that G.C ......-> T.A transversions are the dominant base substitution point mutations observed in the case of (+)-trans-BPDE- ${ }^{2}$-dG adducts [13]. On the basis of these results, we postulate that the normal $C$ still pairs preferentially with the trans-(+)-BPDE-modified $G$ on the template strand. However, among the other three mismatched bases, the probability of A-pairing with the modified $\mathrm{G}$ is highest, thus preferentially inducing G.C -.---> T.A transversions. These considerations provide interesting new possibilities for exploring the molecular mechanisms of mutations induced by other bulky polynuclear aromatic compounds as well. 
In the case of the (-)-trans-BPDE- $\mathrm{N}^{2}-\mathrm{dG}$ adducts, there is no reliable experimental information regarding mutagenic specificities, since mutation experiments with pure (-)-BPDE have not yet been carried out. Based on an examination of the data in Tables $I$ and II, it appears that the values of $\Delta \mathrm{H}^{\circ}$ are highest when $\mathrm{X}=\mathrm{T}$; the corresponding value of $\Delta S^{\circ}$ is the lowest among the three mismatched duplexes. Thus, the situation is analogous to the case of $(+)$ trans adducts, except that $\mathrm{X}=\mathrm{T}$ for the (-)-trans adduct, while $\mathrm{X}=\mathrm{A}$ in the case of the (+)-trans adduct: the most negative value of $\Delta H^{\circ}$ is also paralleled by the most negative value of $\Delta S^{\circ}$ when $X=T$ (Tables I and II). We postulate that the (-)-trans-BPDE- $\mathrm{N}^{2}$-dG adduct preferentially gives rise to G.C -.....-> T.A transversions as in the case of $(+)-$ trans-BPDE- $\mathrm{N}^{2}-\mathrm{dG}$ adduct.

In summary, the thermodynamic melting profile experiments with mismatched bases, provide a working hypothesis which can account for the dominance of G.C .......> T.A transitions in the case of (+)-BPDE; this prediction is ronsistent with the experimentally observed mutational specificity of (+)-BPDE [13]. Furthermore, this hypothesis predicts that the preferred base substitution of the (-)-enantiomer of BPDE will be G.C -..-.-.> A.T transitions.

This latter hypothesis remains to be verified.

\section{The Stabilizing Effect of Bulges: Possible Mechanisms of Frameshift Mutations.}

Frameshift mutations occur when one or more base in a sequence are "missed" during replication: the polymerases skip over one or more base to produce deletions as shown in the following example, where $Y$ stands for any of the four bases:

replication

\section{....CCTAAAYYCCCCCGGT.. ...................> ....GGATTT*GGGGGCCA..}

In this example two bases are missed, and thus the replicated strand on the right side lacks the two complementary bases to $\mathrm{YY}$ at the starred site (-2 frameshift mutation). Recently, there have been some exciting developments concerning the mechanisms of frameshift mutations induced by the mutagen and carcinogen acetylaminofluorene (AAF) [14]. One mechanism for -1 frameshift mutations in which only one base is deleted from the replicated strand, involves the formation of a bulge in the modified single strand as follows:

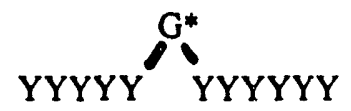


In this rather simplified view taken from reference 14 , the growth of the nascent strand is slowed at the modified base $\mathrm{G}^{*}$ and the polymerase eventually skips over and ignores the $\mathrm{G}^{*}$ base. thus producing a replicated strand missing one $\mathrm{C}$ (deletion of on base). This mechanism is especially favored in sequences in which there are runs of several G's on the 5 '-side of $\mathrm{G}^{*}$ because of "strand-slippage" [14].

Such a mechanism might be favored in the case of BPDE- $\mathrm{N}^{2}-\mathrm{dG}$ adducts as well, particularly if the existence of the BPDE-dG lesions in bulged conformations is energetically feasible. In order to explore this possibility, we have synthesized a complementary strand $5^{\prime}-\mathrm{d}\left(\right.$ GGTAGGATGG) $-3^{\prime}$ for the modified sequence $5^{\prime}-\mathrm{d}\left(\mathrm{CCATCG}{ }^{\mathrm{BPDE}} \mathrm{CTACC}\right)-\hat{j}$ ' described above. We have annealed the two strands to form the duplexes $V$, which are expected to form a bulge as shown below:

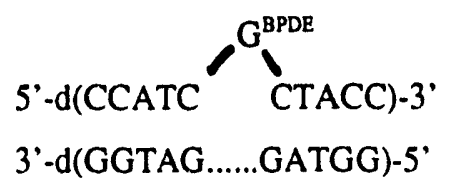

(modified duplex with bulge, No. V)

The thermal stability of this duplex was compared with that of the umodified analog:

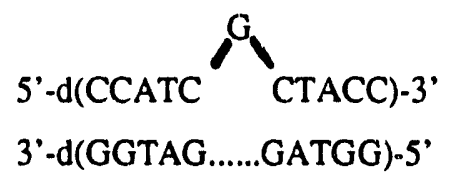

(unmodified duplex with bulge, No. VI)

Since the complementary strand in VI is lacking one base the duplexes V and VI are expected to be much less stable than the corresponding modified and unmodified duplexes I and III, respectively, in which the complementary strand contains all 11 bases. Up till now, we have not yet completed the rather extensive thermodynamic studies from which the molar enthalpies and entropies of duplex formation can be evaluated. However, preliminary results on melting profiles, summarized in Table IV below, show that there is a dramatic stabilizing effect of the BPDE residue.

Table IV. Melting points of modified (V) and unmodified (VI) bulged duplexes.

\begin{tabular}{|c|c|c|}
\hline & $\mathrm{T}_{\mathrm{m}},{ }^{\circ} \mathrm{C}$ & \%Hyperchromicity \\
\hline unmodified (VI) & 21.0 & $12 \pm 2$ \\
\hline (+)-trans adduct (V) & 37.1 & $15 \pm 2$ \\
\hline (-)-trans adduct (V) & 39.5 & $13 \pm 2$ \\
\hline
\end{tabular}


The unmodified bulged duplex has a melting point of only $21^{\circ} \mathrm{C}$ which is some 30 degrees lower than the melting point of $50.5^{\circ}$ observed in the case of the unmodified duplex III in which all 11 base-pairs are hydrogen-bonded (Table III). Thus, the absence of just one base in the complementary strand in duplex VI, has a dramatic effect on its melting point! While this effect is entirely expected, the striking stabilizing effects of the BPDE- $\mathrm{N}^{2}-\mathrm{dG}$ lesion in the (+)-trans and (-)-trans adducts is not. The presence of these BPDE residues raises the melting points of the duplexes from 21 to $37-40^{\circ} \mathrm{C}$; these latter melting points are equal to or higher than those measured with the mismatched duplexes II, in which there are 11 bases in the complementary strand with only one of them being different from the natural complement base (Table III). In fact, judging from the $T_{m}$ values, the bulged duplexes $V$ are only slightly less stable than the mismatched duplexes II (compare the entries in columns 2 in Tables III and IV). The extraordinary stabilities of the bulged modified duplexes $V$ suggests that such structures, in which the BPDE-bearing guanine is swung away from the neighboring bases, might be indeed important in replication. The hyperchromicity values, which are an indication of base-base stacking interactions, are also greater in the modified than in the unmodified duplexes. These reesults show that bulged BPDE carcinogen-DNA structures are thermodynamically feasible, and are thus worthy of further study.

\section{E. SYNTHESIS OF SITE-SPECIFIC AND STEREOSPECIFIC BPDE-OLIGONUCLEOTIDE ADDUCTS WITH MULTIPLE ADJACENT GUANINES.}

Considerable evidence has been accumulated that runs of guanines constitute preferential, though not exclusive mutational hot-spots (see, for example, 13 and 14, and references therein). Up till now, we have described experiments with modified oligonucleotide strands which contained only one guanine. Since the diol epoxide BPDE reacts mostly with the exocyclic amino group of guanine, we found that the preparation of such adducts using direct synthesis methods was rather straightforward $[2,3]$. These methods involve the chemical binding reaction of BPDE with $G$ in synthetic oligonucleotides in aqueous solution. The BPDE-modified oligonucleotides are separated from the unmodified oligonucleotides and subsequently purified by high performance liquid chromatography methods. The modification of oligonucleotides bearing one or more guanines is also rather straightforward, but two problems must be resolved : (1) the aqueous reaction mixtures now contain more than one major adduct, corresponsding to the chemical binding of BPDE to each guanine, and (2) the site of BPDE-binding in the oligonucleotide sequence must be determined.

Both of these problems have been resolved (publication No. 6, see citation at the end of this report). The purification problem was solved by adjusting the solvent elution conditions in both gradient and isocratic HPLC modes. The problem of identifying the particular modified guanine in an oligonucleotide that contains multiple guanines has also been resolved by Maxam-Gilbert high resolution gel electrophoresis methods (publication No. 6). These techniques 
are someivhat too lengthy to be described here. Details are either about to be published (publication No. 6), or will be published within the next year (two papers, based on the material summarized below, are in preparation).

In our research, dealing with base-sequence effects in the mutagenesis induced by bulky polynuclear aromatic compounds, we are collaborating with Dr. E. Loechler of the Biology Department, at Boston University. Dr. Loechler is an expert in site-directed mutagenesis [15]. In this technique, a short oligonucleotide bearing a stereochemically defined covalently bound BPDE residue is inserted into a plasmid by recombinant DNA trechniques; this modified plasmid is then allowed to replicate in bacterial cells, and the types of mutations induced are examined by DNA sequencing methods. In contrast, in random mutagenesis experiments, the BPDE (or other alkylating agent) is added to suspensions of plasmids and allowed to react to form covalent DNA adducts; the positions of the modified gaunines in the plasmids are not determined in this type of experiment. The mutation spectrun, after expression in bacterial cells, is examined and the DNA sequences most prone to mutations are determined. Recently, Dr. Loechler has carried out random-mutagenicity experiments in which he found several sequences which are hot-spots for mutations induced by (+)BPLE. Two of these sequences are the following:

$$
\begin{array}{ll}
5^{\prime}-\mathrm{d}\left(\mathrm{TACG}_{1} \mathrm{G}_{2} \mathrm{CCA}\right)-3^{\prime} & \text { (IAS sequence) } \\
\left.5^{\prime}-\mathrm{d}\left(\mathrm{G}_{1} \mathrm{CG}_{2} \mathrm{G}_{3} \mathrm{CCAAG}\right)_{4}\right)-3^{\prime} & \text { (NAS sequence) }
\end{array}
$$

The subscripts serve as labels for the different guanines, while the abbreviations IAS and NAS are used at Boston University to designate these two different sequences. In sequences IAS and NAS, the guanines $G_{1}$ and $G_{2}$, respectively, are the preferred sites of mutation. We have synthesized (+)-trans-BPDE- $N^{2}$-dG adducts at positio: $\mathrm{G}_{1}$ and $G_{2}$ (IAS), and guanines $G_{2}, G_{3}$ and $G_{4}$ (NAS). These modified DNA sequences were supplied (9/92) to Dr. Loechler, who will be performing site-directed mutagenicity experiments. In parallel, we will be characterizing these same sequences in the single-stranded and double-stranded forms in an effort to determine whether the structural characteristics of the adducts, the perturbations of the local DNA structure, and the thermodynamic characteristics of the duplexes provide any insight into the molecular mechanisms underlying the mutagenicities of these isomeric (+)-trans-BPDEoligonucleotide adducts.

Finally, in order to study the properties of (+)-trans-BPDE adducts situated in runs of guanines, we have matified the following sequence with (+)-BPDE:

$$
5^{\prime} \cdot \mathrm{d}\left(\mathrm{CTATG}_{1} \mathrm{G}_{2} \mathrm{G}_{3} \mathrm{TATC}\right) \quad \text { (GGG sequence) }
$$


The covalent (+)-trans-BPDE-N2-dG adducts are situated at either of the three guanines. The separation of the adducts was achieved by two successive HPLC elution protocols. The final HPLC elution profile, showing three prominent elution peaks, each containing one of the three adducts, is shown in Fig. 4. The adducts were detected via their absorbances at $260 \mathrm{~nm}$ and $350 \mathrm{~nm}$. Only the BPDE-chromophore-bearing oligonucleotides give rise to absorbance signals at $350 \mathrm{~nm}$, as shown in the absorytion spectra on the left (the top panel depicts the absorption spectrum of the unmodified oligonucleotide d(CTATGGGTATC)). The positions of the modifications on either of the three guanines $G_{1}, G_{2}$, or $G_{3}$, was determined by gel electrophoresis Maxam-Gilbert selective strand scission methods (publication No. 6).

\section{CONCLUDING REMARKS}

We have developed the necessary techniques for synthesizing and purifying BPDE-guanine adducts placed at specific positions in runs of up to three guanines. We believe that the synthesis of such stereospecific and site-specific model carcinogen/mutagen-DNA duplexes represents significant progress towards the ultimate goal of elucidating the molecular mechanisms of mutations induced by bulky polynuclear aromatic compounds, and the associated base-sequence effects. A variety of physico-chemical, structural, and biological studies can be carried out utilizing such site-specific. and stereospecific mutagen-DNA model sequences. These studies include site-directed mutagenesis, in vitro mutagenesis, solution structural studies by NMR, and thermodynamic studies of base-pairing and mispairing. Such studies, only in their initial stages at this time, are showing great promise for relating chemical structural adduct characteristics to their biochemical, and ultimately to their biological characteristics.

\section{Program for the Next Year.}

Basically, there are no significant alterations in our research program for next year.

Our studies next year can be divided into several different categories:

(1) Thermodynamic studies of base-sequence effects on the characteristics of BPDE-modified oligonucleotides with nearcomplementary opposite strands, as discussed in this Progress Report. First, the studies described here will be completed. Second, studies using the sequences IAS, NAS, and the triple G sequence (GGG, above) will be pursued with the (t)trans-BPDE- $\mathrm{N}^{2}-\mathrm{dG}$ adducts situated at each of the multiple G's. These results will be correlated with biological mutation specificity data which will be forthcoming from Dr. Loechler's laboratory at Boston University. 


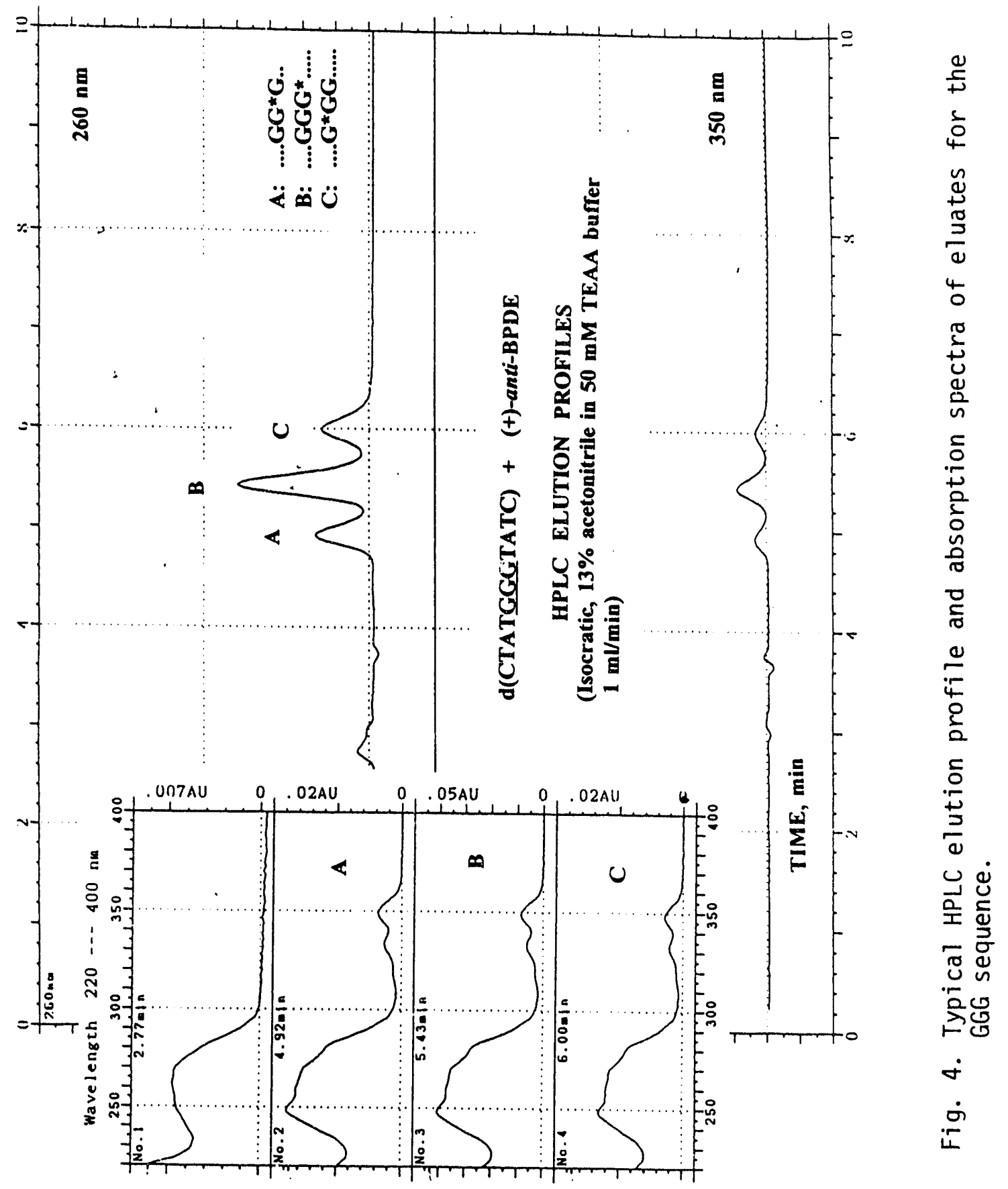


(2) The characteristics of bulge formation, and the reasons for the stabilities of such bulges containing covalently bound $(+)$ - and (-)-trans-BPDE residues will be explored using complementary sequences which are lacking either one (as described here), or two bases opposite to the lesion.

(3) We are in the process of synthesizing oligonucleotides in which the target bases are adenines rather than guanines. The BPDE-adenine $\left(\mathrm{N}^{6}\right)$ adducts are expected to exhibit conformations in which the BPDE residue is located in the major groove, rather than in the minor groove (as in BPDE- $\mathrm{N}^{2}$-dG adducts).

(4) Our other major research theme involves studies of base-sequence on reactivities of diol epoxide derivatives such as BPDE, and the conformations of the adducts formed. Of particular interest are the effects of stereochemistry which strongly affect the conformations of the adducts formed [3,5,6]. In this project, we use an approach in which the effects of base-sequence on adduct structures are examined by a build-up technique: the adduct structural characteristics are examined in dinucleotides, trinucleotides, tetranucleotides, etc., in which the nature of the bases flanking the BPDEmodified base is varied. These studies are also in progress, and will be fully described in our next report.

\section{REFERENCES}

1. A.H. Conney, Cancer Res. (1982) 42, 4875-4917.

2. M. Cosman, V. Ibanez, N.E. Geacintov and R.G. Harvey (1990) Carcinogenesis 11, 1667-1672.

3. N.E. Geacintov, M. Cosman, B. Mao, A. Alfano, V. Ibanez and R.G. Harvey (1991) Carcinogenesis, 12 , 2009-2018.

4. S.C. Cheng, B.D. Hilton, J.M. Roman and A. Dipple (1989) Chem. Res. Toxicol. 1, 334-340.

5. M. Cosman, C. de los Santos, R. Fiala, B.E. Hingerty, S.B. Singh, V. Ibanez, L.A. Margulis, D. Live, N.E. Geacintov, S. Broyde anó D.J. Patel (1992) Proc. Natl. Acad. Sci. (USA) 89, 1914-1918.

6. C. de los Santos, M. Cosman, B.E. Hingerty, V. Ibanez, L.A. Margulis, N.E. Geacintov, S. Broyde and D.J. Patel (1992) Biochemistry 31, 5245-5252.

7. M. Cosman et al., in preparation.

8. Harvey, in Polycyclic Hydrocarbons and Carcinogenesis, R.G. Harvey (ed.) ACS Symposium Series, 1985, pp. 3562.

9. L.A. Marky, L. Canuel, R.A. Jones and K.J. Breslauer, Biophys. Chem. (1981) 13, 141-149.

10 B.L. Gaffney and R.A. Jones, Biochemistry (1989) 28, 5881-5889.

11. M. Warshaw, C.R. Cantor and I. Tinoco, in Handbook of Biochemistry and Molecular Biology, 3rd ed., Fasman, G. (ed.), CRC Press. Cleveland, OH, pp. 589-595.

12. L.A. Marky and K. Breslauer, Biopolym. 26 (1987) 1601-1620. 
13. Mckay, W., Benasutti. M., Drouin, E. and Loechler. E. (1992) Carcinogenesis 13, 1415-1425.

14. I.B. Lambert, R.L. Napolitano and R.P.P. Fuchs (1992) Proc. Nall. Acad. Sci. USA 89, 1310-1314.

\section{PUBLICATIONS ACKNOWLEDGING SUPPORT BY DOE GRANT DE-FG02-88ER60647}

January 1, 1991 - August 31, 1992

1. "Structures of the (+) and (-)-trans-anti-BPDE adducts to guanine-N2 in a duplex dodecamer." S.B. Singh, B.E. Hingerty, U.C. Singh, J.P. Greenberg, N.E. Geacintov and S. Broyde, Cancer Res., (1991) 51, 3482-3492.

2. "Dependence of conformations of benzo[a]pyrene diol epoxide-DNA adducts derived from stereoisomers of different tumorigenicities on base sequence." C.J. Roche, A.M. Jeffrey, B. Mao, A. Alfano, S.K. Kim., V. Ibanez and N.E. Geacintov, Chem. Res. Toxicol. 4, (1991), 311-317.

3. "Spectroscopic characteristics and site I/site II classification of cis and trans benzo[a]pyrene diolepoxide enantiomerguanosine adducts in oligonucleotides and polynucleotides." N.E. Geacintov, M. Cosman, B. Mao, A. Alfano, V. Ibanez and R.G. Harvey (1991) Carcinogenesis 12, 2099-2018.

4. "Photoinduced electron transfer and fluorescence mechanisms in covalently linked polynuclear aromatic nucleotide complexes." N.E. Geacintov, B. Mao, L.L. France, R. Zhao, J. Chen, T.-M. Liu, N.-Q. Ya, L.A. Margulis, and J.C. Sutherland (1992), S.P.I.E. Proceedings, Time-Resolved Laser Spectroscopy in Biochemistry III, Vol. 1640, 774-783.

\section{IN PRESS (8/31/92)}

5. "Thermodynamics of helix-coil transitions in stereospecific benzo[a]pyrene diol epoxide-oligonucleotide addıcts." M. Cosman, N. E. Geacintov, and S. Amin, Polycyclic Aromatic Hydrocarbons, 1992.

6. "Direct synthesis and identification of benzo[a]pyrene diol epoxide-deoxyguanosine binding sites in modified oligodeoxynucleotides. B. Mao, L.A. Margulis, B. Li, V. Ibanez, H. Lee, R.G. Harvey and N.E. Geacintov (1992) Chem. Res. Toxicol. 5, 000-000. 
7. "Base-sequence dependence of covalent binding of benzo[a]pyrene diol epoxide to guanine in oligodeoxyribonucleotides." L. A. Margulis, V. Ibanez and N. E. Geacintov, Chem. Res. Toxicol. 

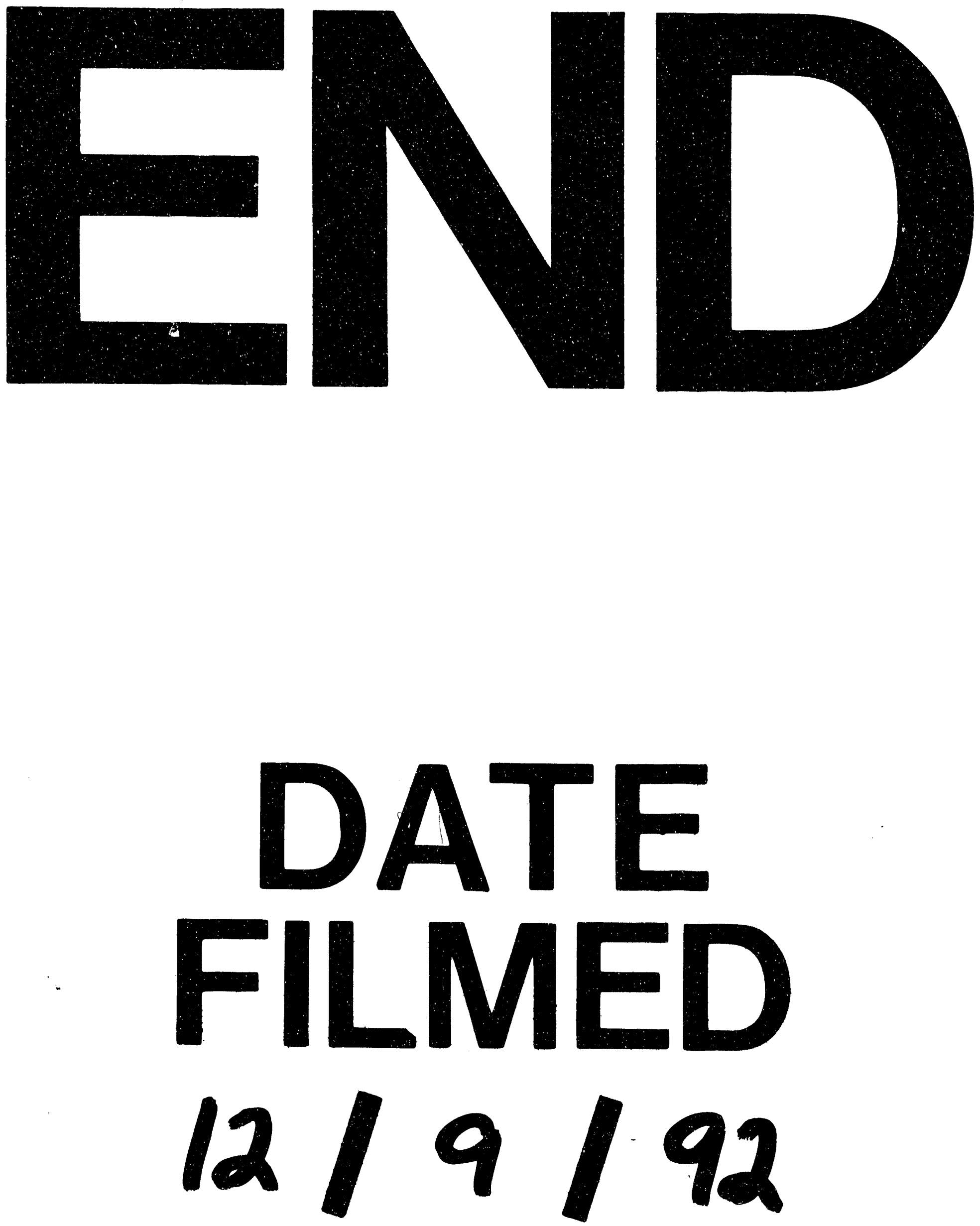


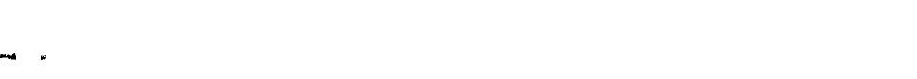

